\title{
Proposta de um instrumento de avaliação da saúde do idoso institucionalizado baseado no conceito do Conjunto de Dados Essenciais em Enfermagem*
}

\author{
A proposal for a health evaluation tool for the institucionalized aged based on the \\ concept of Nursing Minimum Data Set \\ Propuesta de una herramienta de evaluación de la salud del anciano institucionalizado basado en \\ lo concepto del Conjunto de Datos Esenciales de Enfermería
}

\author{
Rita de Cássia Ribeiro', Heimar de Fatima Marin' \\ 'Universidade Federal de São Paulo. Departamento de Enfermagem. São Paulo, SP
}

Submissão: $11 / 03 / 2008$

Aprovação: 24/02/2009

\section{RESUMO}

Este artigo tem como objetivo apresentar o instrumento de avaliação de saúde do idoso institucionalizado desenvolvido com a utilização do conceito de Conjunto de Elementos Essenciais de Enfermagem dividido em duas seções: identificação e avaliação de saúde. Trata-se de uma pesquisa metodológica sobre o desenvolvimento de um instrumento de coleta de dados. O instrumento constitui-se de duas seções: Seção A com as informações de identificação na admissão, e a Seção B é o Formulário de Avaliação Total, contendo dados dos padrões cognitivos, de comunicação, audição, visão, humor e comportamento e condições fisiológicas e nutricionais. Conclui-se Que este instrumento é uma ferramenta capaz de agregar os dados mais importantes para subsidiar a assistência de enfermagem na atenção ao idoso institucionalizado.

Descritores: Coleta de dados; Registros de enfermagem; Informática em enfermagem; Sistemas de informação; Enfermagem geriátrica.

\begin{abstract}
This article aims at presenting a health evaluation tool for the institutionalized aged developed under the concepts of the Nursing Minimum Data Set (NMDS) divided in two sections: identification and health evaluation. It is a methodological research about the development of a data collection tool. The tool is constituted of two sections: Section A contains identification data in the admission, and Section B is the Total Assessment Form, containing data of cognitive, communication, hearing, vision, mood and behavior patterns, and physiological and nutritional conditions. It was concluded that this tool is capable of aggregating the most important data to subside nursing assistance in the care of the institutionalized aged.

Descriptors: Data collection; Nursing records; Nursing informatics; Information systems; Geriatric nursing.
\end{abstract}

\section{RESUMEN}

Este artículo tiene como objetivo presentar una herramienta de evaluación de la salud del anciano institucionalizado basado en lo concepto de lo Conjunto de Datos Esenciales de Enfermería dividido en dos secciones: identificación y evaluación de salud. Tratase de una investigación metodologica sobre el desarrollo de una herramienta de recolección de datos. La herramienta es constituida de dos secciones: Sección A con las informaciones de identificación en la admisión, y la Sección B es el Formulario de Evaluación Total, conteniendo datos de los patrones cognitivos, de comunicación, audición, visión, humor y comportamiento y condiciones fisiológicas y nutricionales. Se hay concluido que esta herramienta es capaz de reunir los datos más importantes para basar la asistencia de enfermería en la atención al anciano institucionalizado.

Descriptores: Recolección de datos; Registros de enfermería; Informática en enfermería; Sistemas de información; Enfermería geriátrica.

* Resultados da Dissertação apresentada ao Departamento de Enfermagem da Universidade Federal de São Paulo para obtenção do título de Mestre em Ciências, em 2007.

Correspondência: Rita de Cássia Ribeiro. Rua Palmares, 672. Brooklin Paulista. CEP 04634-020. São Paulo, SP. 


\section{INTRODUÇÃO}

Atualmente, com a sistematização e informatização da coleta de dados do cuidado de enfermagem, pode-se assegurar a Qualidade da assistência, da administração, da pesQuisa e da fundamentação desse cuidado. A importância do conjunto mínimo (ou essencial) de dados de enfermagem na avaliação da saúde do idoso institucionalizado é Que, com o acesso à informação, o enfermeiro tem a facilidade de planejar e gerenciar o cuidado de enfermagem, favorecendo a avaliação das mudanças no estado dos residentes e proporcionando planos de cuidados adequadamente $\mathrm{e}^{(\mathrm{l})}$.

No campo da enfermagem, a informatização surge como um alicerce para um salto na Qualidade da assistência, gerência e ensino, tornando cada fase do processo interativa e dinâmica. A construção coletiva do conhecimento pode contribuir para identificar melhores formas de fazer as coisas, conscientizando os profissionais e melhorando a Qualidade e a eficiência da prestação dos serviços ${ }^{(1)}$.

Os Sistemas de Cuidado ao Paciente, também chamados de Sistemas de Informação em Enfermagem, devem atender às necessidades dos profissionais Que atuam na assistência direta ao paciente (enfermagem), com o objetivo de construir o prontuário do paciente de modo que o mesmo contenha todos os elementos relacionados aos atendimentos prestados em QualQuer setor e em QualQuer época ${ }^{(1,2)}$.Em termos de conteúdo,os dados são modelados de acordo com as fases do Processo de Enfermagem (PE), Que é um método sistemático e contínuo de planejamento e gerenciamento do cuidado do paciente, oferecendo aos enfermeiros uma maneira lógica e racional de organizar informações, de modo Que o cuidado de enfermagem seja adequado e efetivo. Todas as fases do PE estão inter-relacionadas e compõem um circulo contínuo de pensamento e ação, tendo assim uma característica dinâmica ${ }^{(3)}$. A fase inicial, a coleta de dados, define o conteúdo e o desenvolvimento das fases seguinte, mas deve-se considerar sempre que o dado do paciente possui característica dinâmica, sendo originado em diferentes fontes e formatos, ou seja, são dados médicos, nutricionais, de cuidados e outros, na forma de gráficos, textos, tabelas e imagens, por exemplo ${ }^{(3)}$

Diante da diversidade e de inúmeros exemplos de sistemas de informação de enfermagem, um dos primeiros esforços para estabelecer um padrão uniforme para a coleta de dados de enfermagem foi desenvolvido por Werley et $\mathrm{al}^{(4)}$ em 1988, o denominado Nursing Minimum Data Set (NMDS). Este modelo foi planejado para facilitar a abstração do mínimo, um núcleo comum de dados para descrever a prática de enfermagem ${ }^{(5)}$.

$\mathrm{Na}$ área de avaliação do NMDS Americano na atenção ao idoso institucionalizado, outro modelo desenvolvido foi o Resident Assessment Instrument (RAI), Que é um conjunto de dados multidisciplinar Que contém dados de cuidados de enfermagem à pacientes e clientes em nursing homes, ou seja, em casas de assistência ao idoso. Proporciona uma estrutura e linguagem para o entendimento do cuidado em longo prazo e desenha planos de cuidado, avaliando Qualitativamente, descrevendo a população assistida para o planejamento e política de esforços ${ }^{(6)}$. O RAI, eneuanto sistema, contempla as informações da identificação e fornece dados a respeito de: rotina da pessoa, comunicação dos padrões de audição, funcionamento físico, bem estar psico-social, padrões de atividade, condições de saúde, estado oral ou dental, uso de medicamento, informação anterior, padrões cognitivos, padrões de visão, continência, problemas de humor e comportamentais, diagnostico da doença, estado oral ou nutricional, condição da pele e tratamentos ou procedimentos.

$\mathrm{Na}$ atenção a saúde do idoso, no histórico, o enfermeiro deve procurar evidências de funcionamento anormal ou de fatores de risco Que contribuam para com os problemas de saúde ${ }^{(7)}$. Para isto, torna-se importante o emprego de instrumentos investigativos bem elaborados, capazes de orientar o registro de dados importantes da situação atual do cliente. Aqui é fundamental considerar a Quantidade e a Qualidade da informação disponível. Além disso, encontra-se o profissional diante da enorme diversidade de sintomas e sinais freqüentemente manifestos pelo idoso, Que são particularmente menos característicos e evidentes do Que nos jovens, exigindo constante atenção e percepção a fim de não subestimar os indícios menos implícitos e, por outro lado, valorizar excessivamente aspectos decorrentes do processo natural de envelhecimento. Esses dados formam a base para planejar o cuidado de enfermagem.

Nas experiências compartilhadas na área de saúde do idoso, especialmente no atendimento a idosos institucionalizados, constase Que a coleta de dados na admissão de clientes não é, na maioria das vezes, suficientemente satisfatória para uma análise detalhada de sua condição de saúde. Isto dificulta o planejamento da ação de enfermagem. Não existe, de modo geral, um protocolo para direcionar a assistência, nem tão pouco é assegurado o comprometimento do profissional em atualizar o mínimo de informações necessárias para melhor atender o cliente.

É necessário enfatizar Que a implementação de um conjunto essencial de elementos para a enfermagem requer o uso de uma terminologia comum para a prática de enfermagem, consistente e completa documentação, e uma aplicação de apoio computadorizado para facilitar a documentação, a ligação, o armazenamento e a recuperação de dados. Além disto, o uso de registros computadorizados para prover dados do cuidado de enfermagem é considerado o investimento estratégico para a enfermagem nos próximos anos, o Que decorre o principio "uma vez coletado, muitas vezes utilizados" ${ }^{(8)}$.

Portanto padronizar e identificar o conjunto mínimo de dados Que forneça informação suficiente e necessária na avaliação inicial do idoso é fundamental. Ou seja, os dados coletados nesta fase do processo de enfermagem podem ser padronizados em um conjunto de itens essenciais, Que podem auxiliar o enfermeiro a identificar a saúde global do idoso, e podem oferecer subsídios para Que o enfermeiro tome suas decisões na elaboração de planos de cuidados de enfermagem mais eficazes, melhorando significativamente a Qualidade da assistência à clientela em Questão.

Na tentativa de responder as Questões de ineficiência na coleta de dados na atenção à saúde do idoso, em especial àQueles institucionalizados, este artigo tem como objetivo apresentar e descrever um instrumento de avaliação de saúde do idoso institucionalizado, com base no conceito de NMDS.

\section{METODOLOGIA}

Trata-se de um estudo não-experimental do tipo metodológico(9). O objeto do estudo em Questão é o desenvolvimento e validação 
de um instrumento de coleta de dados voltado especificamente para o idoso institucionalizado. Tais instrumentos podem ser desenvolvidos com base em Qualquer uma das abordagens teóricas ou conceituais de enfermagem, devendo descrever as características do indivíduo e suas respostas ao estado de saúde para atender as necessidades de informação ${ }^{(2)}$.

Com esta perspectiva, utilizou-se como referencial teóricoanalítico o conceito do Conjunto de Dados Essenciais de Enfermagem $^{(4)}$ (NMDS-Nursing Minimun Data Set). Ainda, foi também empregado o modelo conceitual de Wanda Horta ${ }^{(10)}$ Que permite a avaliação do paciente com seus componentes bio-psicosócio-espirituais, e o instrumento $\mathrm{RAI}^{(5)}$ como estrutura referencial para a avaliação inicial do paciente.

O processo de elaboração do instrumento envolveu inicialmente a escolha das subdimensões relacionadas ao estado de saúde global do idoso visando subseqüentemente identificar os respectivos domínios para avaliação. Foi dividido em seções específicas para cada domínio, com o objetivo de facilitar o registro e a visualização dos dados, de forma Que cada dado coletado possa gerar uma informação importante e Que realmente seja utilizada. Estas seções foram delineadas da seguinte forma: Seção A, contendo o domínio Informação de Identificação na Admissão, composta por três subdimensões; Seção B, contendo o domínio Avaliação Total, composta por 14 subdimensões.

O estudo foi submetido e aprovado pelo Comitê de Ética e Pesquisa da UNIFESP sob o protocolo n $0052 / 06$.

\section{RESULTADOS E DISCUSSÃO}

A construção deste formulário foi orientada pelo conceito exposto no NMDS, o Qual apresenta 16 categorias, classificadas aqui em duas grandes seções assim denominadas: Seção A - com os dados de identificação na admissão (Anexo I) e Seção B - contendo o formulário de avaliação total (Anexo 2).

A seção A tem como objetivo registrar informação Que identifique o cliente, o responsável legal e um espaço destinado a outros contatos: são registros nomeados de subdimensões. Al,A2, A3. A padronização destes dados ainda, é considerada um problema devido à grande heterogeneidade nas diferentes instituições. $\mathrm{O}$ objetivo dessa opção é permitir a comunicação com sistemas que utilizam tais características e a disponibilização de uma consulta capaz de encontrar dados da pessoa de forma eficiente. Para o DATASUS consideram-se como características de um sistema de identificação, as informações demográficas como nome, endereço, escolaridade, documentos, idade, entre outras ${ }^{(11)}$.

A Seção B (Anexo 2) é a mais extensa do instrumento e possui 14 subdimensões ou conjuntos de dados: do residente (BI), padrões cognitivos com o Mini-Exame do Estado Mental (B2), indicadores de Delirium (B3), padrões de comunicação/audição (B4), padrões de visão (B5), padrões de humor e comportamento (B6), funcionamento físico e problemas estruturais (Index de Katz) (B7), condições da pele (B8), estado nutricional (B9), eliminação urinária/ intestinal (B|0), medicamentos (B|l), condições de saúde (B|2), padrões de atividade (BI3) e informação da avaliação (B।4).

Todos estes conjuntos de dados têm como objetivo coletar dados essenciais da avaliação global de saúde do idoso, onde são investigados aspectos de sua vida nos níveis bio-psico-sócio- espirituais Oferece dados para identificação de problemas, elaboração dos diagnósticos de enfermagem, uma intervenção individualizada, a evolução e uma análise mais fundamentada das ações de enfermagem. É um formulário extenso, com protocolos para avaliação funcional, cognitiva, comportamental, além de outras subdimensões importantes para a atuação de enfermagem.

Para a subdimensão B2, Padrões cognitivos, foi contemplada a versão do $\mathrm{MEEM}^{(12)}$ (Mini Exame do Estado Mental) protocolo que é utilizado no Brasil, validado por Bertollucci et $\mathrm{al}^{(13)}$. Na área de saúde do idoso, na grande maioria das vezes, está-se diante de Queixas relacionadas à memória, seja pela maior freeüência ou pelo fato de ser a função Que mais preocupam pacientes e familiares, com respeito à possibilidade de evolução para demência. É freQüente o encontro de alterações mentais e comportamentais no idoso. Em face de suspeita de declínio das funções intelectuais, o primeiro procedimento é verificar se tais alterações podem ser relacionadas às habitualmente encontradas no envelhecimento normal.. Este procedimento pode ser realizado pela equipe multiprofissional, não sendo, portanto, exclusivo do enfermeiro ${ }^{(13)}$.

A subdimensão B.3 é referente aos indicadores de delírium. Neste estudo optou-se em utilizar alguns dos sintomas de fácil identificação descritos na literatura e no RAI (Regiment Assesement Instrument), por apresentar uma forma mais simples de entendimento e mais completa eneuanto dado para ser avaliado ${ }^{(6,7)}$.

Delírium é uma Síndrome cerebral orgânica sem etiologia especifica, caracterizada pela presença simultânea de perturbações da consciência e da atenção, da percepção do pensamento, da memória, do comportamento psicomotor, das emoções e do ritmo sono-vigília. É condição fundamental a identificação dos fatores etiológicos envolvidos ${ }^{(14)}$.

As subdimensões B4 e B5, referentes aos padrões de comunicação, audição e visão. Os estados de diminuição da audição e visão e o comportamento da memória e do raciocínio diminuem a comunicação de maneira acentuada ${ }^{(15)}$. Os dados Que compõem esta subdimensão servem de alerta para o Enfermeiro personalizar a assistência no intuito de conservar o sentido de valor e dignidade do paciente. Detectar problemas do individuo, suas duvidas, dificuldades de verbalização, desconforto a visão e afins, podem impedir ou dificultar a independência do idoso na realização das $\mathrm{AVDs}^{(15)}$.Cabe aqui a atuação do enfermeiro na saúde ocular do idoso e na prevenção da cegueira, por meio da investigação e detecção precoce de alterações oculares, para Que as ações de enfermagem favoreçam a independência do idoso e o seu bem estar.

$\mathrm{Na}$ subdimensão B6, padrões de humor e comportamento (indicadores de depressão, ansiedade, mau humor), apresentam-se dados Que auxiliam na identificação de deficiências Que provocam impacto na capacidade global funcional. Pode ajudar o enfermeiro a determinar Quais os comportamentos Que estão prejudicados e ajudam na tomada de decisões, úteis também para avaliar o progresso do cliente em relação às intervenções. De acordo com Roch $^{(16)}$ a constatação de informações Que mensuram o estado afetivo serve para diferenciar entre o tipo de depressão (grave, leve, moderada) Que afeta o funcionamento e a má disposição de espírito, comum a muitas pessoas. Essa situação é muito freeüentemente vinculada à perda, principalmente a afetiva, e as situações relacionadas aos papeis familiares, laborais e sociais. $\mathrm{O}$ objetivo aqui é conhecer o idoso no que diz respeito a estes aspectos 
e planejar uma assistência, respeitando as características de cada individuo. Sabendo-se que o instrumento de admissão em ILPI pode ser concluído pelo enfermeiro em até sete dias, existe tempo suficiente para uma melhor observação destes aspectos ${ }^{(7)}$.

$\mathrm{Na}$ subdimensão B7, Funcionamento físico e problemas estruturais. Estes itens descrevem o nível funcional do individuo no tempo, mede objetivamente os efeitos dos tratamentos Que pretendem restaurar a função, amplamente utilizada para subsidiar o enfermeiro na avaliação do grau de dependência da clientela em Questão. Sabe-se Que o envelhecimento provoca minimização das funcionalidades dos sistemas, diminuição do sistema perceptivo evidenciado pela diminuição da acuidade visual e, sobretudo, o desgaste do aparelho locomotor refletido na cinestesia, desencadeando uma pior condição de perceber o corpo em relação aos objetos e às demais pessoas. Em função deste declínio sensorial e motor, o idoso apresenta dificuldade crescente na realização de atividades cotidianas ${ }^{(15,16)}$. Para esta subdimensão optou-se em utilizar o Índice de Katz, uma escala mais descritiva que avalia desempenho em atividades da vida diária, ou seja, sua capacidade funcional e as divide em rotineiras (tomar banho, vestir-se, ir ao banheiro, continência e alimentação) e instrumentais (usar telefone, preparar comida, fazer compras, locomoção fora de casa, medicação e dinheiro). Classifica os pacientes em independente (I), dependente (D) e necessitado de assistência (A). Também respondida pelo responsável do paciente ou pelo próprio paciente. Um instrumento de fácil aplicabilidade, simples, mas importante para o planejamento de cuidados ${ }^{(16)}$. Fornece ao enfermeiro o grau de dependência Que o idoso apresenta, objetivando melhor a assistência de enfermagem como também a distribuição da equipe assistencial.

A subdimensão B8, Condições de pele. Contém dados da região do corpo humano mais susceptível à formação de feridas ou lesões por pressão, com um escore de classificação, o Que facilita a intervenção e a avaliação do tratamento proposto e contém doze itens (ver anexo 2). Esta Questão é importante neste cenário para determinar a possibilidade de lesões relacionadas com quedas e a capacidade do individuo de efetuar tarefas básicas de cuidados pessoais - a necessidade de avaliação da pele para determinar o risco de ocorrência de ulcera de pressão e as medidas preventivas necessárias bem como avaliar a eficácia da terapêutica instituída. Embora mudanças relacionadas à idade ocorram em Quase todo o sistema corporal, as mudanças Que ocorrem no sistema tegumentário são freqüentemente as mais visíveis porque afetam a aparência externa da pessoa ${ }^{(17)}$.

Na subdimensão B9, estado nutricional, é igualmente importante, pois, Quando não observada, pode comprometer o estado geral do idoso. Por isto é importante a observação da mastigação, condição dos dentes, higiene, perda de peso. Alerta para o enfermeiro na orientação da equipe $\mathrm{e}^{(18)}$.

A subdimensão Bl0, Eliminação urinária/intestinal. O trato urinário é um dos sítios mais comuns de infecção bacteriana no idoso. A infecção do trato urinário é um tema bastante abrangente, pois possui peculiaridades não apenas com relação aos aspectos epidemiológicos, mas também Quanto a sua microbiologia, patogênese, Quadro clínico e até mesmo Quanto a alguns procedimentos realizados na terapêutica.É importante reconhecer os fatores de risco ou as condições associadas com a infecção e procurar corrigi-las ${ }^{(19)}$. Também é um indicador de Qualidade bastante expressivo, tanto na avaliação do atendimento, Quanto das condições de higiene do local Que se encontra o idoso. Com o envelhecimento o aparelho digestório apresenta alterações estruturais, de motilidade e da função secretória , Que variam em intensidade e natureza em cada segmento do mesmo. As conseQüências clinicas dessas alterações são, na maioria dos casos, pouco perceptíveis, mas em seu conjunto, adQuirem importância para a compreensão e reconhecimento de sintomas e para a previsão de alterações na farmacocinética de diversas medicações ${ }^{(19)}$.

A subdimensão Bl l, Medicamentos. Nos idosos, a presença de várias doenças e sintomas os tornam grandes consumidores de medicamentos. Cabe à enfermeira investigar esses hábitos para evitar danos e fornecer orientações adequadas. É de importância para a equipe de assistência ao idoso, os sintomas, o controle de horários, a verificação da ingestão dos mesmos, as datas de validade e a observação de sua eficácia. Alerta para casos de incompatibilidade com a terapêutica instituída ${ }^{(18)}$.

Na subdimensão B 12, Condições de saúde. O enfermeiro coleta dados aqui Que não estão evidentes na admissão, mas Que podem ser importantes na elaboração do seu plano de cuidados.

Na subdimensão B1 3, Padrões de atividade. Para melhor adaptar o idoso em uma ILPI é importante saber dos seus hábitos em casa, o Que gosta de fazer no dia a dia. A interação com os outros residentes é muito importante. Após a admissão é recomendável Que a enfermeira o apresente a alguns idosos, ou ao companheiro de Quarto, mostre os locais de recreação, de lazer, as dependências do local onde ele vai residir. A terapeuta ocupacional e o enfermeiro podem discutir maneiras de proporcionar aos idosos, atividades prazerosas. As atividades lúdicas já fazem parte da maioria das ILPIs ${ }^{(20-22)}$. Terapias alternativas também são muito utilizadas, por exemplo, terapia com animais (Ex: trabalho realizado em uma Instituição da cidade de São Paulo) .

$\mathrm{Na}$ última subdimensão (B।4), Informações de avaliação. Os dados aQui são simples, decorrente do resultado, o enfermeiro pode entender se os dados coletados durante toda a admissão são mesmo reais, ou seja, fidedignos. Ao longo da convivência é Que este processo realmente acontece. Lembrando que o enfermeiro pode realizar todo o processo de admissão em até sete dias, dados podem ser modificados. Uma nova avaliação deste instrumento pode ser realizada a cada Quatro meses ou se houver alguma intercorrência antes deste período. O último dado é referente à assinatura do enfermeiro responsável e a data da avaliação.

\section{CONCLUSÃO}

A elaboração de um instrumento de coleta de dados essenciais de enfermagem, com informações confiáveis, é uma ferramenta importante neste cenário, uma vez Que o número de idosos no Brasil cresce vertiginosamente e as Instituições de longa permanência, se apresentam para o futuro como importante equipamento social de atenção a essa população.

Com o processo de avaliação padronizado, a equipe identifica se as áreas Que necessitam mudanças através de um caminho sistemático, melhorando assim a avaliação dos residentes pela identificação de especificas necessidades, proporcionando ao enfermeiro o ajuste aos planos de cuidados, projetados adequadamente para resolução dos casos com melhor Qualidade 
de atendimento.

$\mathrm{O}$ instrumento projetado e aqui proposto mostrou, de modo geral, de acordo com os resultados de avaliação prévia, ser exęüível, contendo os principais dados a serem coletados e analisados. Assim, considera-se Que foi atendida a intenção deste estudo no Que diz respeito à elaboração do instrumento para favorecer a enfermagem em sua prática profissional, subsidiando também o ensino e a pesquisa em enfermagem na atenção à área da saúde do idoso.

\section{REFERÊNCIAS}

I. Ribeiro RC, Marin HF. Proposal for an essential Nursing Data Set to evaluate the health in home care elderly persons. In: Proceedings of the $9^{\text {th }}$ International Nursing Informatics Congress. 30 may 2 jun 2006. Seul; Korea. Seul (KOR): Nursing Informatics; 2006.

2. Marin HF. Informática em enfermagem. São Paulo: EPU; 1995.

3. Marin HF. Os componentes de enfermagem do prontuário eletrônico do paciente. In: Massad E, Marin HF, Azevedo Neto RS, editores. O prontuário eletrônico do paciente na assistência, informação e conhecimento médico. São Paulo: Disciplina de Informática Médica da FMUSP/NIEn/OPAS/OMS; 2003. p. 73-84.

4. Werley H, Ryan P, Zorn CR. The Nursing Minimum Data Set (NMDS): a framework for the organization of nursing language. In: American Nurses Association - ANA. Nursing Data Systems: the emerging framework. Washington: ANA; 1995.

5. Silveira DT, Marin HF. Conjunto de dados essenciais de enfermagem: construindo um modelo em saúde ocupacional. Acta Paul Enferm. 2006; 19(2): 2 1 8-27.

6. Rantz MJ, Popejoy L, Zwygart-Stauffacher M, Wipke-Tevis, D, Grando, VT. Minimum Data Set and Resident Assessment Instrument. Can using standardized assessment improve clinical practice and outcomes of care? I Gerontol Nurs 1999; 25(6): 35-43; Quiz 54-5.

7. Roach S. Introdução à enfermagem gerontológica: aplicação do processo de enfermagem ao cuidado gerontológico. Rio de Janeiro: Guanabara Koogan; 2003.

8. Marin HF, Rodrigues R, Delaney C, Nielsen G, Yan J, editors. Building standard-based Nursing Information Systems. Washington: Pan American Health Organization/World Health Organization, Division of Health Systems and Services Development; 2002.

9. Lobiondo-Wood G, Haber I. PesQuisa em enfermagem: métodos, avaliação crítica e utilização. $4^{\mathrm{a}}$ ed. Rio de Janeiro: Guanabara Koogan; 200I.

10. Horta WA. O processo de enfermagem. São Paulo: EPU; 1979.

11. Ministério da Saúde (BR). Secretaria Executiva. DATASUS. Informações de saúde. Brasilia: Ministério da Saúde; 2006 [citado 2006 jun 20]. Disponível em: http://www.datasus.gov.br/ DATASUS.

12. Folstein MF, Folstein SE, Mchugh PR. "Mini Mental state". A practical method for grading the cognitive state of patients for the clinician. I Psychiatr Res 1975; 12:18.

13. Bertolucci PHF, Okamoto I, Brucki S, Siviero M, Neto I, Ramos L. Applicability of the CERAD neuropsychological battery to brazilian elderly. Are Neuropsiquiatria 2001; 59(3-A): 532-6.

14. Fabbri RMA. Delirium. In: Freitas E, V-Py L. Tratado de geriatria e gerontologia. Rio de Janeiro: Guanabara Koogan; 2002. p. 168-75.

15. Freitas EV, Miranda RD. Parâmetros Clínicos do Envelhecimento e Avaliação Geriátrica Ampla. In: Freitas EV, Miranda RD. Tratado de geriatria e gerontologia. $2^{\text {a }}$ ed. Rio de Janeiro: Guanabara Koogan; 2006. p. 900-9.

16. Roach S. Aplicação do processo de enfermagem. In: Roach S. Introdução à enfermagem gerontológica. Rio de Janeiro: Guanabara Koogan; 2003. p. 46-55.

17. Roach S. Sistema tegumentário. In: Roach S. Introdução à enfermagem gerontológica. Rio de Janeiro: Guanabara Koogan; 2003. p. 163-74.

18. Diogo MJD. Consulta de enfermagem em gerontologia. In: Papaléo Netto M. Gerontologia. A velhice e o envelhecimento em visão globalizada. São Paulo: Atheneu; 2002. p. 209-2 1 .

19. Roach S. Sistema Genitourinário. In: Roach S. Introdução à enfermagem gerontológica. Rio de Janeiro: Guanabara Koogan; 2003. p. 257.

20. Pavarini SCl. Dependência comportamental na velhice: uma analise do cuidado prestado aos idosos institucionalizados [tese]. Campinas: UNICAMP; 1996.

21. Diogo MID, Yamamoto A. Os idosos e as Instituições asilares do município de Campinas. Rev Latino-am Enfermagem 2002; 10(5): 660-6.

22. Duarte YAO. Família: rede de suporte ou fator estress. A ótica de idosos e cuidadores familiares [tese]. São Paulo: Escola de Enfermagem, Universidade de São Paulo; 2001. 
ANEXO 1

\begin{tabular}{|c|c|c|}
\hline \multicolumn{3}{|c|}{ Seção A - Informações de Identificação na Admissão } \\
\hline \multicolumn{3}{|c|}{ A.1. Informações Gerais } \\
\hline \multicolumn{3}{|l|}{ 1. Nome do Residente: } \\
\hline 2. Data da admissão: & \multicolumn{2}{|c|}{ 3. $\quad$ RG: } \\
\hline 4. Data de nascimento: & \multicolumn{2}{|c|}{ 5. Natural de: } \\
\hline 6. Gênero: ( ) Masc ( ) Fem & \multicolumn{2}{|c|}{ 7. Raça: ( ) Branco ( ) Negro ( ) Pardo } \\
\hline 8. Religião: & \multicolumn{2}{|c|}{ 9. Estado Civil: ( ) Casado ( ) Solteiro ( ) Viúvo } \\
\hline 10. Ocupação Anterior: & \multicolumn{2}{|c|}{ Ocupação Atual: } \\
\hline $\begin{array}{l}\text { 11. Procedente de: ( ) Casa ( ) Lar de Idosos } \\
\text { ( ) Hosp Psiquiátrico ( ) Hospital Geral } \\
\text { ( ) Outros: }\end{array}$ & \multicolumn{2}{|c|}{$\begin{array}{l}\text { 12. Nível de instrução: ( ) Analfabeto } \\
\text { ( ) Fundamental Completo } \\
\text { ( ) Fundamental Incompleto } \\
\text { ( ) Ensino Superior } \\
\text { ( ) Pós-Graduação }\end{array}$} \\
\hline \multicolumn{3}{|l|}{$\begin{array}{l}\text { 13. Fonte de pagamento atual para estadia: } \\
\text { ( ) Seguro Saúde ( ) Não pagante } \\
\text { ( ) Particular total ( ） Particular parcial }\end{array}$} \\
\hline \multicolumn{3}{|c|}{ A.2. Responsável Legal } \\
\hline \multicolumn{3}{|l|}{ 1. Nome do responsável: } \\
\hline 2. Grau de parentesco: & \multicolumn{2}{|c|}{ 3. Data de nascimento: } \\
\hline 4. Profissão & RG: & CIC: \\
\hline \multicolumn{2}{|l|}{ 6. Endereço residencial: } & 7. Complemento: \\
\hline \multicolumn{2}{|l|}{ 8. Endereço comercial: } & 9. CEP: \\
\hline \multirow{2}{*}{\multicolumn{3}{|c|}{$\begin{array}{l}\text { Telefone cCelular: } \\
\text { A.3. Outros Contatos }\end{array}$}} \\
\hline & & \\
\hline \multicolumn{2}{|l|}{ 1. Nome do responsável: } & 2. Grau de parentesco: \\
\hline \multicolumn{2}{|l|}{ 3. Telefone Residencial: } & Celular: \\
\hline \multicolumn{2}{|l|}{ 4. Responsável pelo registro: } & 5. Data: \\
\hline
\end{tabular}

ANEXO 2

\begin{tabular}{|c|c|c|}
\hline \multicolumn{3}{|c|}{ Seção B - Formulário de Avaliação Total } \\
\hline \multicolumn{3}{|c|}{ B.I. Dados do Residente } \\
\hline \multicolumn{3}{|l|}{ 14. Nome do Residente: } \\
\hline 15. Unidade: & 16. Quarto: & 17. Leito: \\
\hline \multicolumn{3}{|l|}{ 18. Hipótese Médica Diagnóstica: } \\
\hline \multicolumn{3}{|l|}{ 19. Data da Avaliação: } \\
\hline \multicolumn{3}{|c|}{$\begin{array}{l}\text { B.2. Padrões Cognitivos (Mini-Exame do Estado Mental) } \\
\text { Atribuir I ponto para cada resposta certa }\end{array}$} \\
\hline \multicolumn{3}{|c|}{ B.2.1. Orientação } \\
\hline 1. Mês atual: & \multicolumn{2}{|c|}{ 2. Nome da Instituição: } \\
\hline 3. Local específico (andar ou setor): & 4. Cidade: & 5. UF: \\
\hline \multicolumn{3}{|l|}{ Total de Pontos: } \\
\hline \multicolumn{3}{|c|}{ B.2.2. Memória Imediata } \\
\hline \multicolumn{3}{|c|}{$\begin{array}{l}\text { 1 1. Fale ao residente: vaso, caneca, tijolo e peça Que o mesmo repita as três palavras imediatamente: } \\
\text { Atribuir I ponto para cada palavra pronunciada corretamente. }\end{array}$} \\
\hline \multicolumn{3}{|c|}{ 12. Total de pontos: } \\
\hline \multicolumn{3}{|c|}{ B.2.3. Atenção e Cálculo } \\
\hline \multicolumn{3}{|c|}{$\begin{array}{l}\text { 1. Faça o seguinte cálculo: } 100 \text { - } 7 \text { cinco vezes consecutivas ou se não conseguir solicite para Que soletre a palavra } \\
\text { MUNDO de trás para frente. Atribua OI ponto para cada cálculo correto OU OI ponto pra cada letra soletrada } \\
\text { corretamente. }\end{array}$} \\
\hline \multicolumn{3}{|c|}{ 2. Total de pontos: } \\
\hline \multicolumn{3}{|c|}{ B.2.4. Evocação } \\
\hline \multicolumn{3}{|c|}{$\begin{array}{l}\text { 1. Pergunte pelas } 03 \text { palavras ditas anteriormente: vaso, caneca, tijolo. Atribua OI ponto para cada palavra evocada } \\
\text { corretamente. }\end{array}$} \\
\hline 2. Total de pontos: & & \\
\hline
\end{tabular}


B.2.5. Linguagem

I. Nomear relógio

2. Nomear caneta:

3. Total de pontos:

4. Solicite que o residente repita: "Nem aqui, nem ali, nem lá" (atribuir I ponto para cada repetição correta)

5. Total de pontos:

B.2.6. Solicite Que o residente escreva uma frase (atribuir I ponto se a frase estiver escrita corretamente) Total de pontos:

B.2.7. Solicite ao residente que copie o desenho abaixo. Atribua OI ponto se estiver desenhado corretamente. 1. Total de pontos (Score):

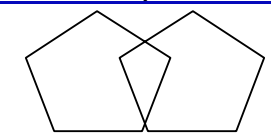

1. Total de pontos (Score):

1. Menor que 24 boa escolaridade / Possível demência ( )

2. Menor Que 18 escolaridade média / Possível demência ( )

3. Menor que 14 analfabeto / Possível demência ( )

\begin{tabular}{|c|}
\hline B.3. Indicadores de Delírium \\
\hline 1. Facilmente distraído ( ) \\
\hline 2. Períodos de letargia ( ) \\
\hline 3. Períodos de percepção alterada ou consciência do que está ao seu redor ( ) \\
\hline 4. Períodos de fala desorganizada ( ) \\
\hline 5. Períodos de inquietação ( ) \\
\hline 6. Funcionamento mental varia ao longo do curso do dia ( ) \\
\hline B.4. Padrões de Comunicação / Audição \\
\hline 1. Audição: Normal ( ) Diminuída ( ) Zumbido ( ) Aparelho auditivo ( ) \\
\hline 2. Clareza da fala: Fala clara ( ) Disartria ( ) Afásico ( ) \\
\hline 3. Capacidade de entender os outros: Entende ( ) Algumas vezes entende ( ) Raramente entende ( ) Não entende ( ) \\
\hline B.5. Padrões de Visão \\
\hline 1. Acuidade visual para a idade: Normal ( ) Alterada ( ) Qual alteração: \\
\hline 2. Aparelhos visuais: Normal ( ) Alterado ( ) Qual alteração: \\
\hline
\end{tabular}

2. Aparelhos visuais: Normal ( ) Alterado ( ) Qual alteração:

B.6. Padrões de Humor e Comportamento

(Indicadores de depressão, ansiedade e mau humor)

1. Expressões verbais de sofrimento:

Falas negativas ( ) Sentimento de inutilidade ( ) Perguntas repetitivas ( ) Culpa inadeeuada ( )

Expressão do Que pode ser um medo real ( ) Pensamentos recorrentes de morte ( )

2. Problemas do ciclo do sono: Mau humor pela manhã ( ) Insônia ou hipersonia aproximadamente todos os dias ( )

3. Triste, Apático, Aparência Ansiosa: Expressões faciais de tristeza, dor, preocupação ( ) Choro, lágrimas ( )

Agitação ou retardo psicomotor ( )

4. Perda do Interesse: Afastamento das atividades de interesse ( ) Interação social diminuída ( )

5. Sintomas Comportamentais: Diminuição da capacidade de pensar ( )

Diminuição da capacidade de concentrar-se ( )

Diminuição da capacidade de tomar decisões ( )

Fala excessiva ( )

Movimento excessivo ( )

Atividade sexual excessiva ( )

Comportamento social inapropriado ( )

Auto-estima exagerada ( )

Irritabilidade ( )

B.7. Funcionamento Físico e Problemas Estruturais (Índex de Katz)

A - Necessita de auxílio para realização de atividades de vida diária.

B - Necessita de auxílio parcial para realização de AVDs.

C - Não necessita de auxílio para as AVDs.

B.7.I. Transferência

1. Entra e sai da cama sozinho, senta e levanta da cadeira sem auxílio $(\mathrm{A} / \mathrm{B} / \mathrm{C})(\mathrm{)})$

2. Entra e sai da cama e/ou cadeira, não faz uma ou mais transferências $(\mathrm{A} / \mathrm{B} / \mathrm{C})(\mathrm{)}$ 
B.7.2. Ir ao toalete

1. Vai ao banheiro, senta e levanta do vaso, arruma as roupas, se limpa $(\mathrm{A} / \mathrm{B} / \mathrm{C})(\mathrm{C})$

2. Usa fraldas ou comadre/papagaio ou recebe assistência para ir ao banheiro e usá-lo $(\mathrm{A} / \mathrm{B} / \mathrm{C})(\mathrm{)}$

B.7.3. Continência

1. Controla completamente urina e fezes $(\mathrm{A} / \mathrm{B} / \mathrm{C})(\mathrm{)}$

2. Parcial ou totalmente incontinente para urinar e evacuar $(\mathrm{A} / \mathrm{B} / \mathrm{C})(\mathrm{)}$

B.7.4. Vestir

1. Pega as roupas no armário, sapatos e acessórios $(\mathrm{A} / \mathrm{B} / \mathrm{C})(\mathrm{)}$

B.7.5. Banho $(\mathrm{A} / \mathrm{B} / \mathrm{C})(\mathrm{)}$

B.8. Condições da Pele

A - Pele íntegra. B - Pele íntegra com áreas hiperemiada. C - Rompimento da pele, bolha. D - Rompimento de pele expondo tecido subcutâneo. E. Rompimento de pele expondo músculo, osso ou tendões.

1. Cabeça $(\mathrm{A} / \mathrm{B} / \mathrm{C} / \mathrm{D} / \mathrm{E})(\mathrm{)}$

2. Pescoço (A/B/C/D/E) ( )

3. Tronco $(\mathrm{A} / \mathrm{B} / \mathrm{C} / \mathrm{D} / \mathrm{E})()$

4. Região sacra (A/B/C/D/E) ( )

5. Maléolo direito $(\mathrm{A} / \mathrm{B} / \mathrm{C} / \mathrm{D} / \mathrm{E})(\mathrm{)})$

6. Maléolo esquerdo $(\mathrm{A} / \mathrm{B} / \mathrm{C} / \mathrm{D} / \mathrm{E})()$

7. Cotovelo direito $(\mathrm{A} / \mathrm{B} / \mathrm{C} / \mathrm{D} / \mathrm{E})(\mathrm{)})$

8. Cotovelo esquerdo $(\mathrm{A} / \mathrm{B} / \mathrm{C} / \mathrm{D} / \mathrm{E})($

9. Região trocanteriana direita $(\mathrm{A} / \mathrm{B} / \mathrm{C} / \mathrm{D} / \mathrm{E})(\mathrm{)}$

10. Região trocanteriana esQuerda $(\mathrm{A} / \mathrm{B} / \mathrm{C} / \mathrm{D} / \mathrm{E})()$

11. Região auricular direita $(\mathrm{A} / \mathrm{B} / \mathrm{C} / \mathrm{D} / \mathrm{E})(\mathrm{)}$

12. Região auricular esquerda (A/B/C/D/E) ( )

\section{B.9. Estado Nutricional}

B.9. I. Problemas orais:

De mastigação ( ) De deglutição ( ) Dor na boca ( ) Xerostomia total ( ) Xerostomia parcial

( ) Presença de próteses ou pontes móveis ( ) Dentes Quebrados / soltos / cariados

( ) Gengivas inflamadas / gengivas edemaciadas com sangramento

( ) Limpeza diária dos dentes / gengiva ou cuidado oral diário pelo residente ou membro da equipe ( ) Inalterada ( )

B.9.2. Peso e altura: Altura estimada: $\quad$ Peso estimado:

B.9.3. Acesso nutricional:

Parenteral ( ) Sonda de alimentação ( ) Sonda nasoenteral ( ) Jejunostomia ( ) Gastrotomia ( )

Uso de técnicas assistivas (copo com sugador, colheres com suporte etc) ( ) Normal (prato) ( )

\section{B. 10. Eliminação Urinária / Intestinal}

B. 10.1. Urinária: Normal ( ） Incontinência urinária ( ) Sonda vesical de demora ( ) Cateterismo intermitente ( ) Fralda ( )

B.10.2. Intestinal:

Eliminação intestinal 1 vez ao dia ( ) Eliminação intestinal 2 vezes ao dia ( ) Eliminação intestinal 3 vezes ao dia ( ) Diarréia (acima de 3 episódios de fezes amolecidas ao dia) ( ) Constipação ( ) Impactação fecal (fecaloma) $($ )

\begin{tabular}{|c|}
\hline $\begin{array}{r}\text { B.1 l. Medicamentos } \\
\end{array}$ \\
\hline 1. Medicamentos usados nos últimos 30 dias: Não ( ) Sim ( ) Quais: \\
\hline $\begin{array}{c}\text { B.12. Condições de Saúde } \\
\text { (problemas apresentados nos últimos } 7 \text { dias, não contemplados nas seçôes anteriores) }\end{array}$ \\
\hline 2. Sim ( ) Quais: \\
\hline B.12.1. Acidentes: \\
\hline 1. Caiu nos últimos 7 dias: Sim ( ) Não( ) \\
\hline 2. Fratura do Quadril: Sim ( ) Não ( ) \\
\hline 3. Outra fratura: Sim ( ) Não ( ) \\
\hline B.13. Padrões de Atividade \\
\hline B.I3.I. Se o residente for comatoso, pule essa seção. \\
\hline B.13.2. Preferência de atividade em geral (adaptado para a capacidade atual do residente) \\
\hline 1. Baralho / outros jogos ( ) 2. Artesanato ( ) 3. Música ( ) 4. Leitura ( ) \\
\hline 5. Assistir TV ( ) 6. Conversar ( ) 7. Passeios ( ) 8. Bingos ( ) \\
\hline 9. Ajudar os outros ( ) 10. Bazar ( ) I1. Atividade espiritual / religiosa ( ) \\
\hline
\end{tabular}




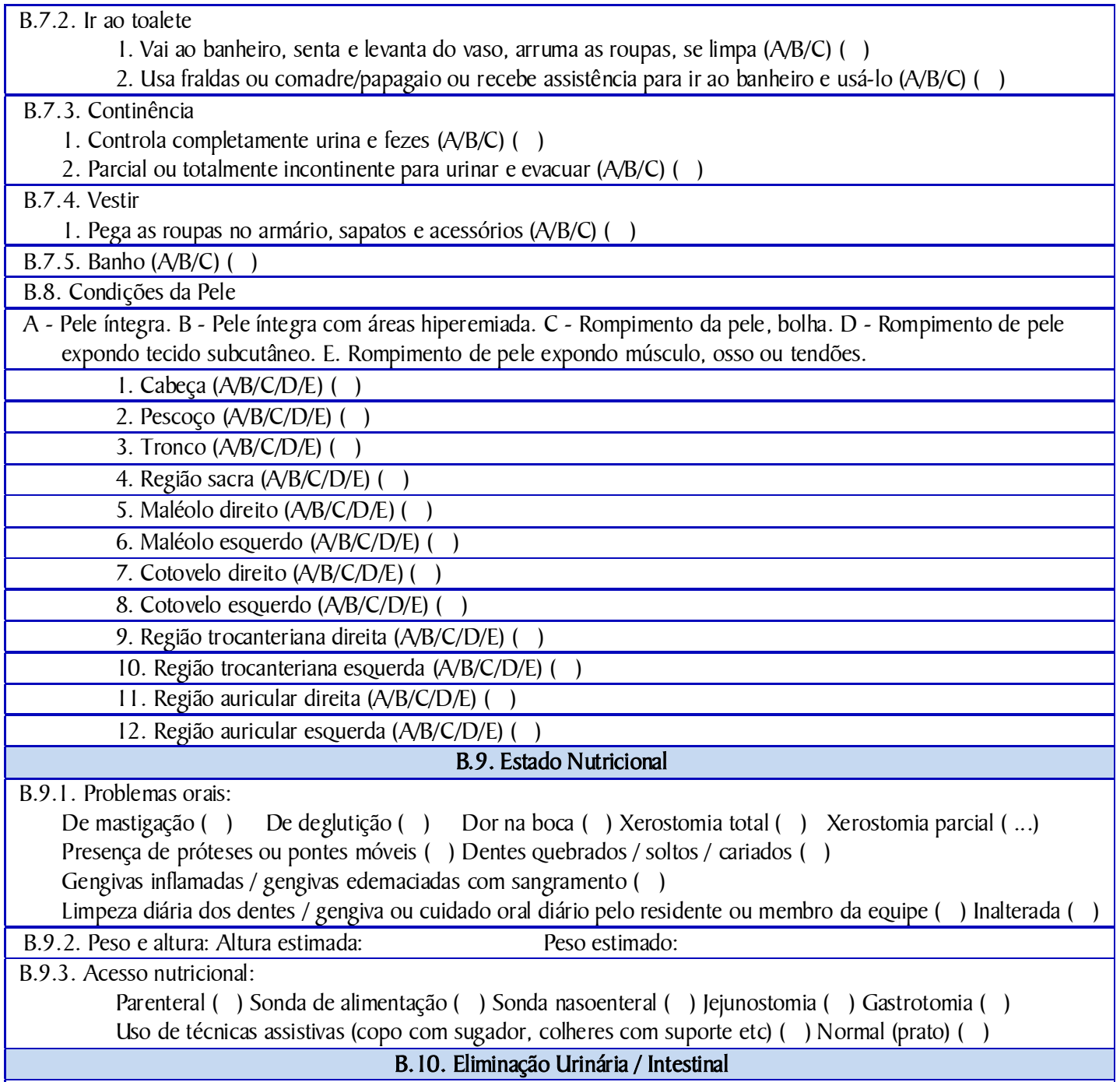

B. I 0.1. Urinária: Normal ( ) Incontinência urinária ( ) Sonda vesical de demora ( ) Cateterismo intermitente ( ) Fralda ( )

B.1 0.2. Intestinal: Eliminação intestinal I vez ao dia ( ) Eliminação intestinal 2 vezes ao dia ( ) Eliminação intestinal 3 vezes ao dia ( ) Diarréia (acima de 3 episódios de fezes amolecidas ao dia) ( ) Constipação ( ) Impactação fecal (fecaloma) ( )

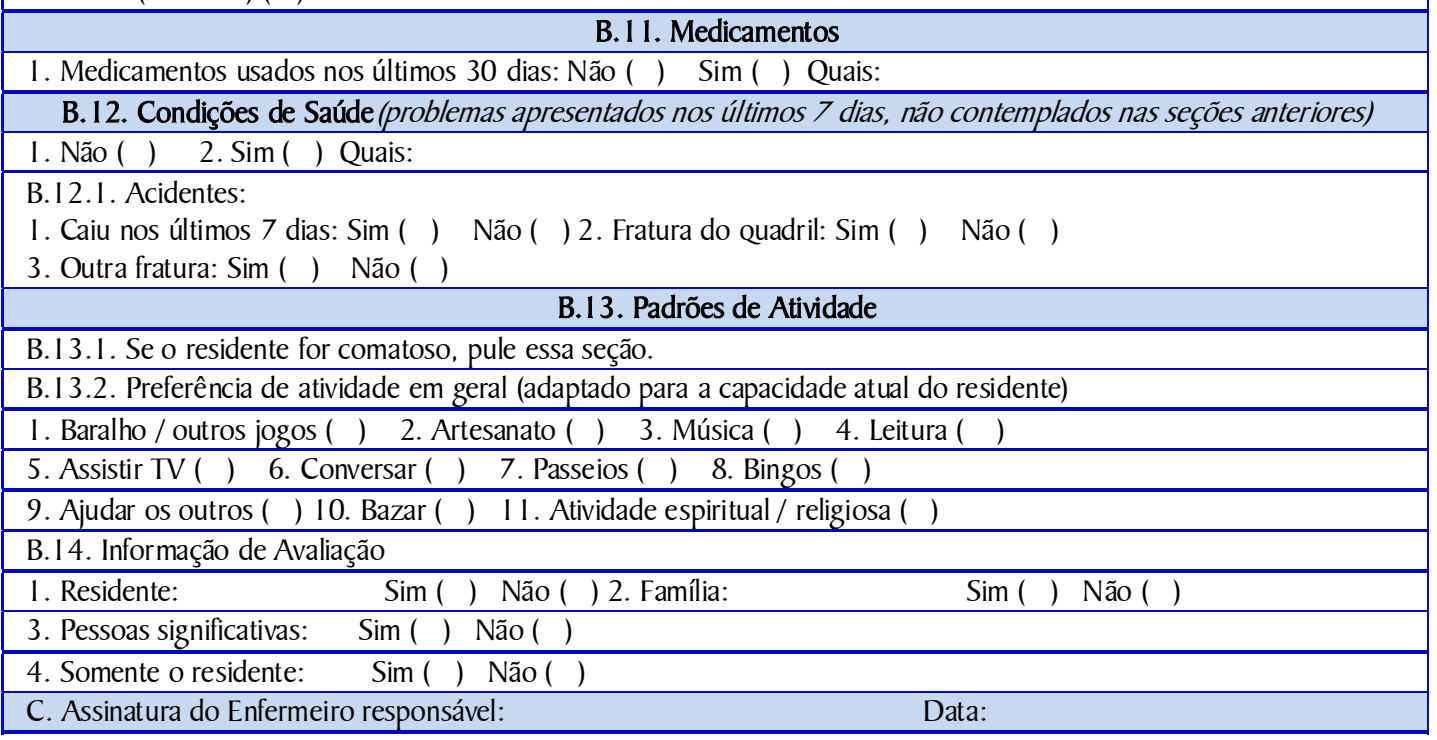

